\title{
Design of Wearable Interface Considering Touch Communications
}

\author{
Mariko Kato and Naoki Saiwaki \\ Guraduate school of Humanities and Science, Nara Women's University, \\ Kitauoya-cho, Nara-shi, Nara. 630-8334, Japan \\ \{ebm.katou, saiwaki\}@cc.nara-wu.ac.jp
}

\begin{abstract}
Wearable computing has the image of the high-level information processing which uses the small-sized computer. However, a motion of the body is barred by the complexity of wiring or apparatus and the function of assistance of operation which is the function of original dress is disregarded in many cases. Moreover, it tends to be kept at a distance that externals look like the cyborg by a general person. On the other hand, the interface that uses the movement of the body recently is actively researched. Then, the prototype system of a good design and fit was developed for the college woman and evaluated in this research. The system has the touch interfaces that pay attention to touch communications and can do the music sharing and the voice conversation by two-way, and the switch can be done by touch.
\end{abstract}

Keywords: Touch Communication, Wearable Interface.

\section{Introduction}

Wearable computing tend to be researched and developed focused on functionality to be able to carry advanced information devices such as a computer, a display, input devices and so on. But on the other hand, the original functions of clothes such as comfortability or supporting of the movement were considered less serious. For example, the motion of the body was limited by the complexity of wiring or attaching devices. Moreover, daily fashion characteristic is tending to be neglected. The appearance to install the wearable equipment is like the strange cyborg. It is not so matured to unite refined design and advanced function yet. In addition, it is a problem that the degree of freedom of the arrangement of the wearable equipments like various interfaces is extremely low. The research that uses electroconductive clothes instead of wiring is paid attention to improve these problems recently [2].

But, the input or operational device which reflects the movements of a body, like a remote controller of Nintendo Wii, is paid attention in resent years. These interfaces enable intuitive and intelligible system operation. From the viewpoint of interface research, it is important that development of the easy operation interface for wearable system based on the idea that why and how we can or should wear these devices. It is thought that not only the miniaturization of device of personal computer but also the development of the interface with the special function that can be realized because the 
equipment is installed in the body is important from the viewpoint of the interface research in the wearable computing.

Then, in this study, we designed the control interface for wearable system focusing a body touch communication [1]. Prototype systems were developed as two clothes and evaluated. These have good and comfortable design for Japanese women's university student, and can switch music sharing or voice conversation between 2 persons automatically with touch pose.

To construct the prototype system based on this idea, we studied from three angles.

1. Consideration of the touch communications

2. Hearing survey of fashion sense of general women's university student for easily accepting and smarter installing arrangement of various devices

3. Evaluation of electronic conductive textile (e-textile) as new material for constructing the interface

It is expected that the e-textile solves the problems of complexity of the wiring and improves appearances and comfortability as clothes.

\section{Touch Communication}

Wearable computing treats a machine very close. From the viewpoint of user interface it is important to think to operate a machine more intuitively. Basically, the present input interface of wearable system is similar to usual PC's such as how to press a key of the specific function. But it is possible to construct better and easy-to-use interface for the users if our purpose and situation of using such system are limited. By using wearable interface installed in clothes and extracting the meaning of user's action such as gesture, attitude and touch by person and person in communication, more effective interaction of wearable system will be realized. There are some researches that the feature of the gesture and the operation is analyzed as the vital signal and the image information for utilizing more intimate interface $[3,4]$. In this research, we focus on development of the interface of body touch communication between 2 persons.

The human knows to live in this world by sense of touch first. Then, the human grows up with abundant haptic stimuli from infant to adults, and learns human relation and fundamental confidence for the neighboring world [5]. The expectations, the excitement feeling and the feeling of fullness that the tactile stimuli bring cannot be replaced by other senses. The custom of the body contact, for instance, frequency, the place, and the meaning are different according to the culture. However, the hand, the shoulder, the forehead, the head, the neck, and the forearm are the contacted places in many cases [6].

Then, we picked up and thought about the following often cases.

1) You can share your intention or feeling by the continuance of touch like handshake.

2) You can show your intention or feeling by the changing position or frequency or strength of touch like hitting the shoulder or high touch.

In case 1 , the touch is very similar to information sharing. So, we designed an example of music sharing function by the conductive clothes. If conductive fabric can be used for making clothes instead of a conduction line, the lead of a portable music 
player becomes unnecessary. In case 2 , such actions mean the switch the information or the mode. For example, the person when speaking, touching the shoulder, pulls note. We thought about effective use when two people get on a motorcycle. When a person who rides back touches the waist of driver they can share music, next when he touches the shoulder of driver they can talk through a microphone. We developed the prototype wearable interface which can switch music sharing and conversation by touch place.

\section{Sharing Information by touch}

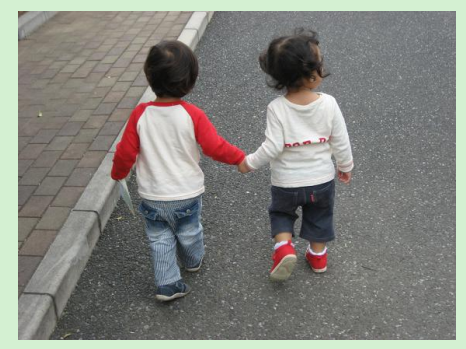

Dress with e-textile can be carried out instead of a conduction line. The lead of a portable music player becomes unnecessary.

You can sharing music with friends, by holding hands, folding arms, etc.

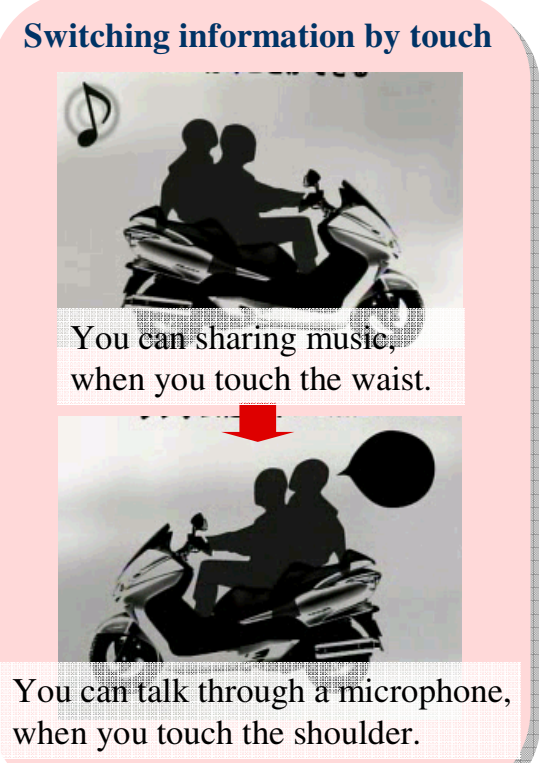

when you touch the shoulder.

Fig. 1. The example of Touch communication

\section{Fundamental Examination for Touch Interface}

\subsection{Appearance Attitude Survey about Wearable Interface Position}

The improvement of the fashionability is one of the problems of the wearable computing for the wide acceptance. This time, if a wearable equipment of how much size was arranged at which position on clothes, it was investigated whether general college women may put on the clothes.

A round sticker was passed to ten testers (college woman in her twenties). The sticker was considered to be a wearable equipment, and pasted on the surface of women's M size jacket. The number of the stickers and places and lab hour were not limited. 


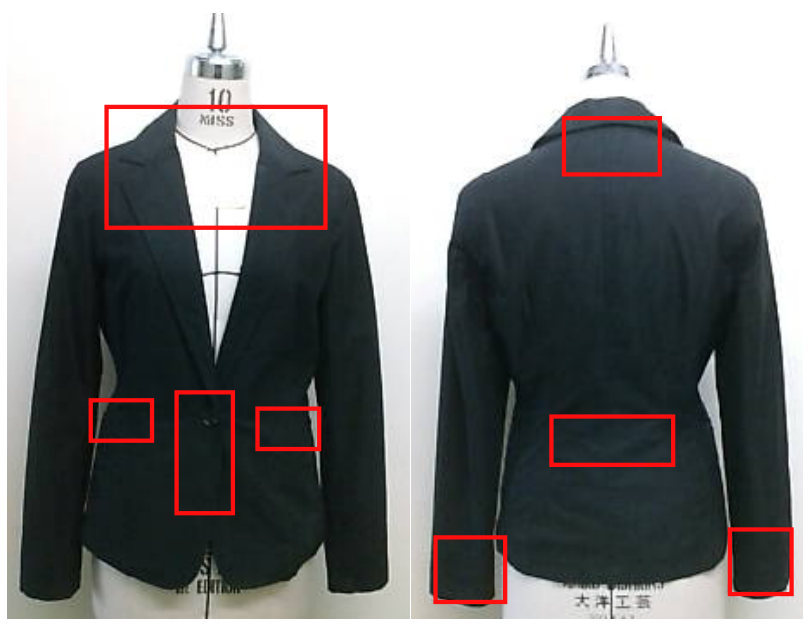

Fig. 2. Place where two people or more pasted sticker

The place where two people or more put the sticker on Fig.2 is shown with red frames. The most popular place is the neck and is the basic place where decoration such as brooches is put. Most people like the design which does not give strange feeling as clothes which installed wearable equipment. From this survey, red frame places of Figure 2 were decided as the equipment installation position of our wearable system. These fixing places were the results of corresponding to the body area where the touch communications were done, and supporting the interface construction possibility by the touch communications. Moreover, it is also possible to put out the equipment to the surface of clothes if accepted as a design.

\subsection{Electrical Specification of e-Textiles}

The cloth made with the fiber with conductivity is called electronic conductive textile (e-textile). Those can because instead of a conduction line or a circuit board. Therefore, the applicability to wearable computing of these is easily expected. However, because the electrical specification of the electroconductive cloth is various depending on the material and the manufacturing method of the cloth, it is necessary to select according to the purpose.

Therefore we measured the electrical resistance and the frequency response of the e-textiles. Three kinds of e-textiles which were sold as cloth for an electromagnetic wave shield were used as an experiment sample. We experimented on three kinds of e-textiles as 1, 2 and 3 respectively (Table. 1).

\subsubsection{Measurement of the Electric Resistance for Alternating (AC) Signal Current}

First we examined the electrical resistance that was the most basic characteristic. We cut e-textiles into 2 rectangles of $2 \mathrm{~cm} * 60 \mathrm{~cm}$ and $30 \mathrm{~cm} * 60 \mathrm{~cm}$ respectively as the samples. The size of the cutting e-textile $(30 \mathrm{~cm} * 60 \mathrm{~cm})$ was almost the half of body 
of a garment in consideration of making the female clothes with the electroconductive

Table 1. Specification of three kinds of e-textiles

\begin{tabular}{|l|l|l|}
\hline 1 & 2 & 3 \\
& & \\
& & \\
\hline \begin{tabular}{l|l|} 
Base material: nylon knitted \\
PA6-20f1 denier
\end{tabular} & $\begin{array}{l}\text { Base material: } \\
87 \% \text { polyester knit } \\
\text { Silver coating (purity }<99.9 \%)\end{array}$ \\
Heat resistance: $-30 \sim 90$ degrees & $13 \%$ carbon fiber \\
\hline \begin{tabular}{l|l} 
Weight: \\
$35 \mathrm{~g} / \mathrm{m}^{\wedge} 2 \pm 5 \%$
\end{tabular} & $75 \mathrm{~g} / \mathrm{m}^{\wedge} 2 \pm 10 \%$ & $130 \mathrm{~g} / \mathrm{m}^{\wedge} 2 \pm 10 \%$ \\
& & \\
\hline
\end{tabular}

cloth in this experiment. We were aimed at checking the difference of electrical characteristic with the wide shape and the narrow shape that was close to the usual electric conduction line. The resistance between the vicinity of length was measured (three times for narrow sample, five times for wide samples), and the mean value was calculate respectively.

As for the result, electric resistance of the wider is one digit smaller than the narrow. Because e-textiles are weaved by the electroconductive string, the route where electricity flows becomes the net which has many irregularity, and very complex. But generally, resistance decreases gradually as width is expanded though even details were not analyzed this time. Therefore, in usually use, it can be considered as resistance of the parallel connection. Therefore, it is clear that e-textile doesn't have any problem even if it connected direct or alternative current power source.

\subsubsection{Frequency Response}

Then, the frequency response to the alternative signal was examined as follows. It is because the phase and the gain change greatly if the alternative signal of the high frequency is input to the electroconductive material like the net, and there is a possibility of negatively affecting the signal. We prepared 12 samples in total by three kinds of e-textiles by 2 shapes and set by 2 patterns in the following. There are two methods when e-textile is used instead of the wire. One is the parallel array and the other is piling them up. In both case, two e-textiles are not to touch each other.

1) Two e-textiles were separated in $5 \mathrm{~cm}$ and put them in parallel. This corresponded to set e-textiles to one side of dress. 


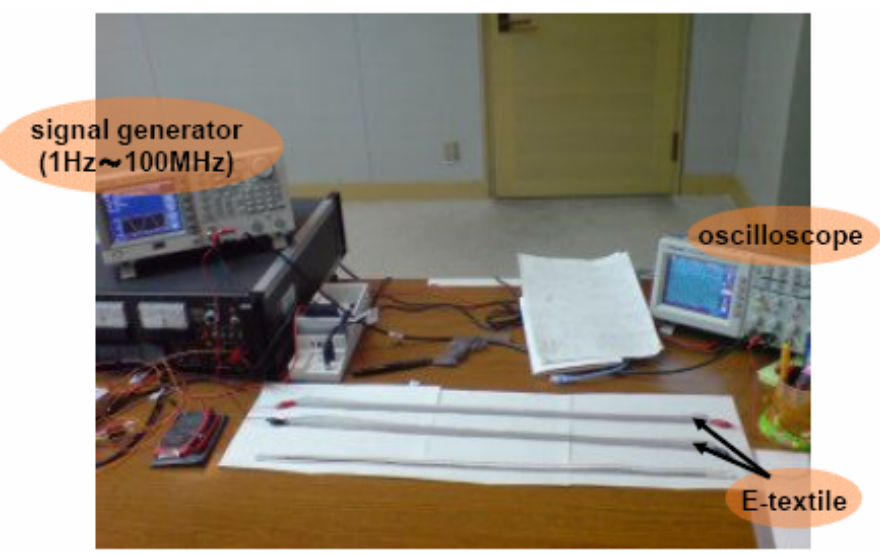

Fig. 3. Frequency response experiment
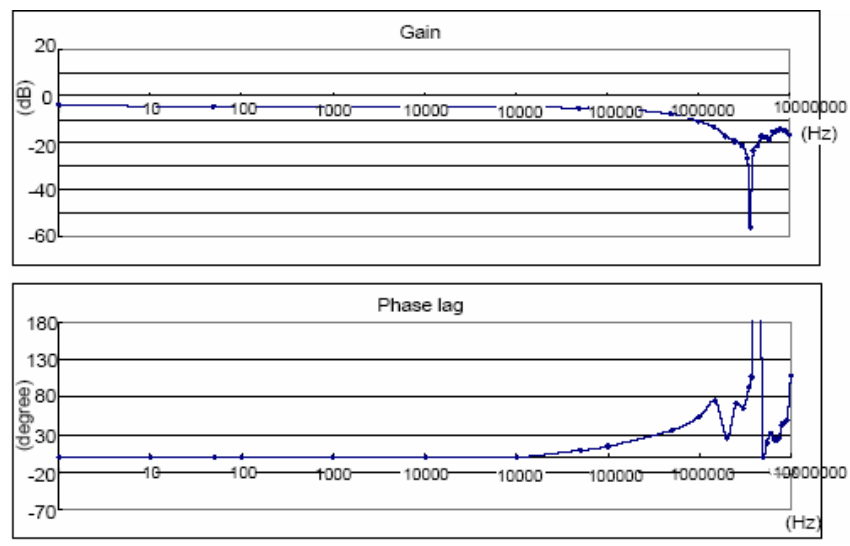

Fig. 4. Gain and phase of signal in each frequency of sample 2

2) Two e-textiles sandwiched the non-conductive textile. This corresponded to set etextiles to both sides of dress like TextileNet system [1].

Two cutting e-textiles used by 3.2.2 were prepared respectively, and the signal of the frequency from $1 \mathrm{~Hz}$ to $100 \mathrm{MHz}$ was input to the e-textile by a signal generator. And, the gain and the phase were calculated from the pair of input/output signals Fig.3).

The result of the gain and the phase are shown in Fig.4 as an example. This sample is e-textile 1, narrow and parallel type. These results are below;

1) Any condition passed the signal through $1 \mathrm{MHz}$ without trouble. It seems that it is possible to use it for the bandwidth of VHF on practical use without trouble.

2) The specific frequency to which the signal extremely attenuates exists though the level is different according to arrangement and shape of e-textiles.

3) When the cloth was piled, the level of the attenuation of the signal was larger than that of the case arranged in parallel. 
First of all, it is possible to use e-textile as wire if the gain and the phase are stable. If the gain and the phase greatly change, the energy of the signal is lost as heat or as the electromagnetic radiation discharged from the e-textile like the antenna. However, the discharge of heat and the electromagnetic radiation were not seen while experimenting. It seems that using such frequency should be avoided anyway. Finally, it becomes a capacitor when the e-textiles are piled up. And there is worry that influences the signal according to the frequency. When the e-textiles are sewn up to clothes, it is necessary to note it. Anyway, it was basically confirmed that electric characteristic of it was no problem in the signaling property by our preliminary experiment.

\section{Construction and Evaluation Experiment of the Prototype System}

Based on the examination described by Chapters 2, 3, we made prototype wearable interface system which can switch music sharing to conversation by touch.

\subsection{Construction of the Prototype System}

We made two cloths including e-textiles and equipments. Fig.5 and Fig.6 show that whole of the prototype system.

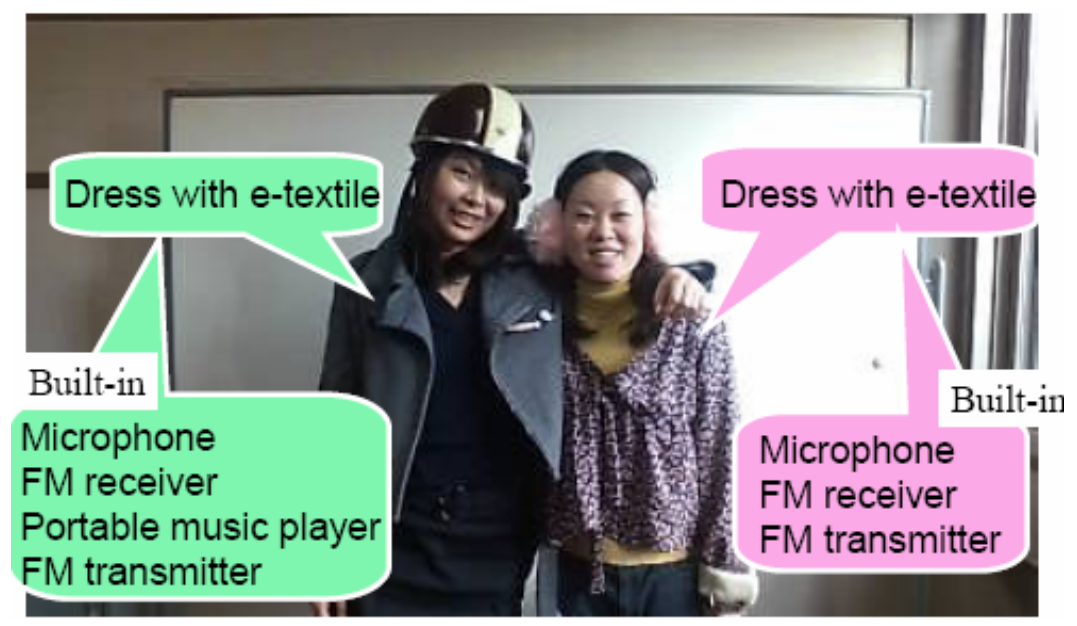

Fig. 5. The prototype system whole

In rider's jacket of gray on the left in the Fig.5 and Fig.6, e-textile is sewn in inside the arm, side and the shoulder, and everything has been connected electrically. In shirt of pink on the right in the Fig.5 and Fig.6, e-textile is sewn in inside the arm, the cuffs and the shoulder, and it is connected electrically from the cuff to the shoulder and divided by a left body and a right body. 


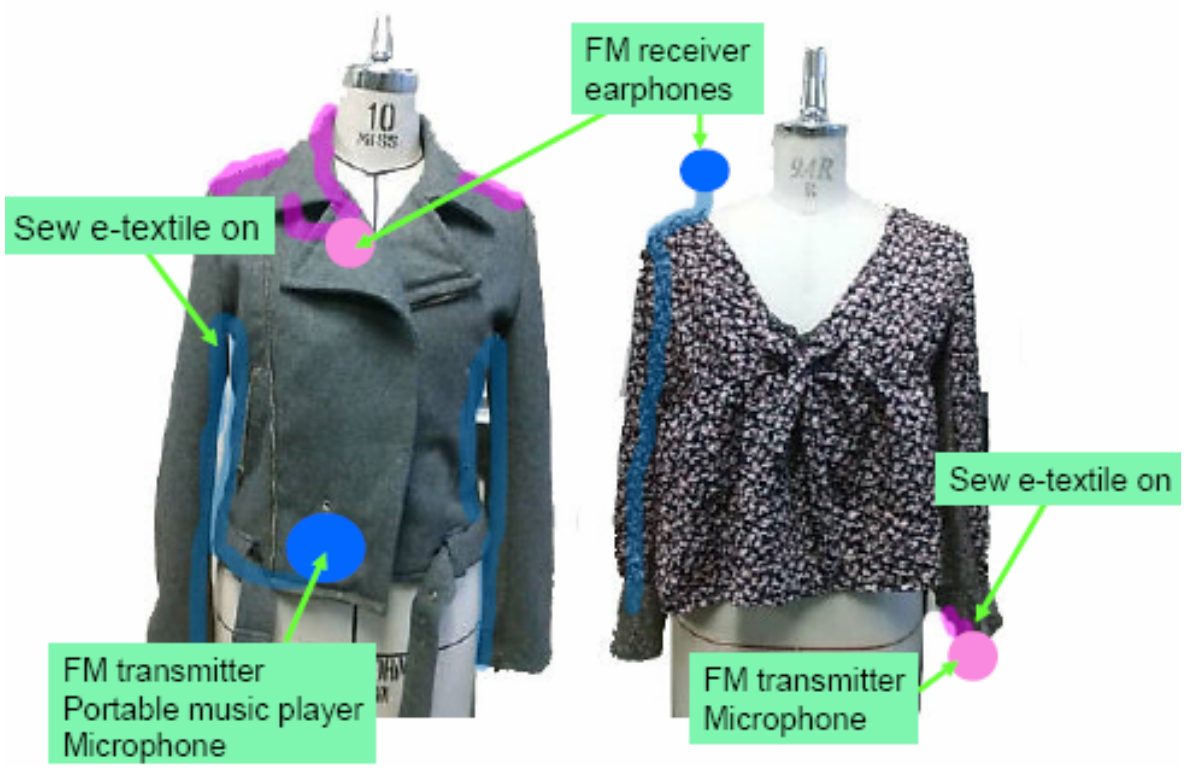

Fig. 6. The prototype system whole
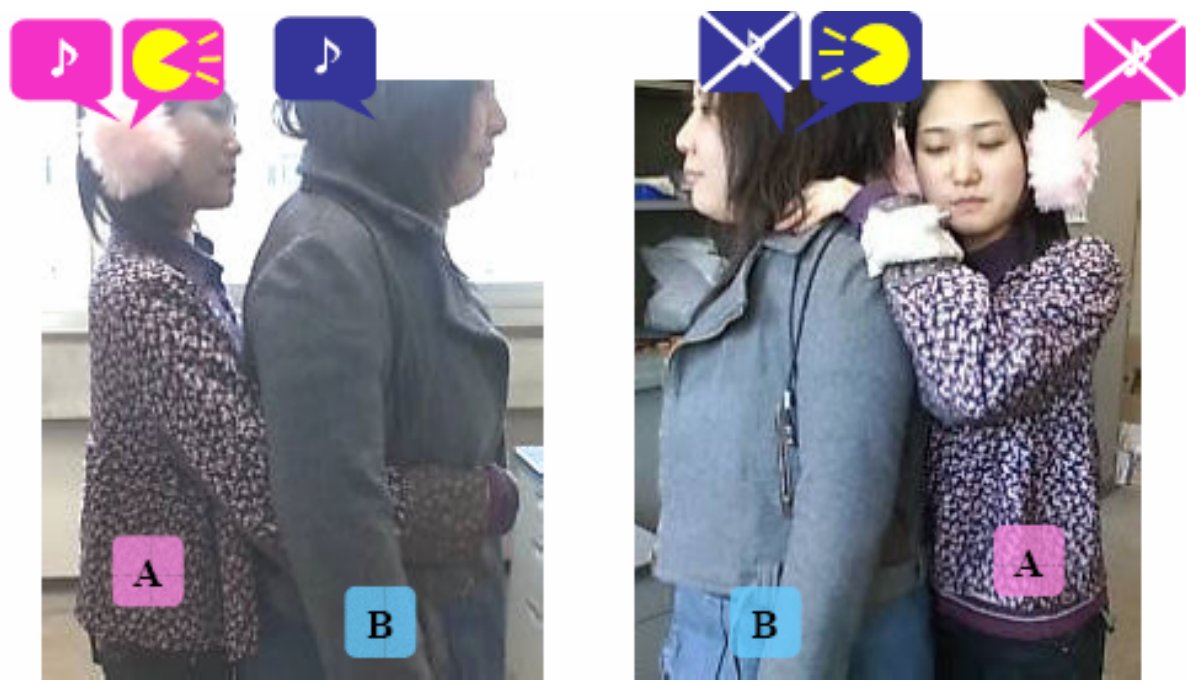

Fig. 7. Example of touch communications by prototype system

It designed like clothes marketed for the college woman so as not to consider user's wearing a special, wearable device to the body. The portable music player and FM transmitter were put in the pocket of a left rider's jacket. Music is transmitted through the e-textile. The user who put on the rider's jacket can listen to music with FM receiver installed on the neck. On the other hand, the user who put on a right floral print 
shirt can share the same music by touching the rider's jacket. Because both clothes are connected by the e-textile, and the signal from the rider's jacket is received from the cuff and transmitted to the shoulder. And, FM receiver and the earphone enter a pink earmuffs and music is played. It is a situation riding the motorcycle by two people while listening to music.

On the other hand, the microphone and FM transmitter are buried under the white scrunchy applied to the left hand of the floral print shirt, and the user can be switched to the conversation mode by touching the shoulder of the rider's jacket. It is operation to touch other's shoulder to take the other's attention. The microphone is installed in the helmet of the rider's jacket user also, and rider's voice is transmitted to the floral print shirt. Fig. 7 shows the example of such a touch communications.

\subsection{Evaluation Experiment of the Prototype System}

We did the evaluation experiment that used prototype system. Four general women's university students as subjects used the system in pairs. And we ask them for feedback as to the system freely.

There was an opinion that it wanted to use this system to share feelings and the topic like not only the motorcycle travel but also the haunted house and the movie theater and so on, in the place where it was difficult to speak. The following answers were in the good point that the subjects had pointed out.

1. The evaluation on the interface side: Operation that moves the hand to the shoulder when speaking connects operation with the meaning knowing by intuition and is comprehensible.

2. The evaluation as a wearable device: Neither the size nor the wiring for the equipment are not anxious.

3. The evaluation of externals: the design is natural and is lovely. Using the e-textiles as an accent is cool.

Therefore, effectiveness in the touch communications interface that had been constructed this time was shown. There was also negative opinion that the operation with touch is hard to understand, and operating the traditional flip switch is easier to use. There was other negative opinion about unstableness of the touch operation it self and the bad of the sound quality.

\section{Conclusion and Future Work}

In this study, we studied from three angles.

1. Wearable interface based on touch communication.

2. Smart and refined design for arrangement of various devices.

3. Experiment on electrical resistance and a frequency response of the e-textile.

In this research, the interface which could switch the function by the touch communication were proposed and developed.

Other various possibilities are thought by the touch interface. The interface of the new kind of thinking also has the problem that the user is not accustomed to use it. However, every user can recognize the effectiveness of the touch communication interface in short time and be accustomed. 
On the other hand, there is a limit in our system that uses an analog circuit like this time for the accuracy of touch and the improvement of the function and tone quality. The digitalization of the system is scheduled to be examined in the future.

\section{References}

1. Kato, M., Terada, T., Akita, J., Toda, M., Saiwaki, N.: Design of Wearable Interface considering Touch Communications. In: Human Interface Society of Japan symposium 2008, pp. 879-880 (2008)

2. Akita, J., Shimmura, T., Murakami, T., Toda, M.: Flexible Network System for Wearable Computing. Transactions of Information Processing Society of Japan 47(12), 1-10 (2006)

3. Fukumoto, M.: Whisper: A Wristwatch Style Wearable Handset. In: Proc. of CHI 1999, pp. 112-119 (1999)

4. Kume, Y., Shimada, M.: A Wearable Input Device Using Wristwatch-like Camera and Reflection Marker. The Journal of The Institute of Image information and Television Engineers 60(2), 249-253 (2006)

5. Iwamura, Y.: Touch; Igaku-Shoin (2001)

6. Nomura, M.: Miburi to shigusa no jinruigaku [Anthropology of a body language and gesture]; Chuokoron-Shinsha (1996) 